\title{
Intravitreal Injection of the Attenuated Pseudorabies Virus PRV Bartha Results in Infection of the Hamster Suprachiasmatic Nucleus Only by Retrograde Transsynaptic Transport via Autonomic Circuits
}

\author{
Gary E. Pickard, ${ }^{1}$ Cynthia A. Smeraski, ${ }^{1}$ Christine C. Tomlinson, ${ }^{1}$ Bruce W. Banfield, ${ }^{3}$ Jessica Kaufman, ${ }^{1}$ \\ Christine L. Wilcox, ${ }^{2}$ Lynn W. Enquist, ${ }^{4}$ and Patricia J. Sollars ${ }^{1}$ \\ Departments of ${ }^{1}$ Anatomy and Neurobiology and ${ }^{2}$ Microbiology, Colorado State University, Fort Collins, Colorado 80523, \\ ${ }^{3}$ Department of Microbiology, University of Colorado Health Sciences Center, Denver, Colorado 80262, and ${ }^{4}$ Department \\ of Molecular Biology, Princeton University, Princeton, New Jersey 08544
}

Intravitreal injection of the attenuated strain of pseudorabies virus (PRV Bartha) results in transneuronal spread of virus to a restricted set of central nuclei in the rat and mouse. We examined the pattern of central infection in the golden hamster after intravitreal inoculation with a recombinant strain of PRV Bartha constructed to express enhanced green fluorescent protein (PRV 152). Neurons in a subset of retinorecipient nuclei [i.e., suprachiasmatic nucleus (SCN), intergeniculate leaflet, olivary pretectal nucleus (OPN), and lateral terminal nucleus] and autonomic nuclei [i.e., paraventricular hypothalamic nucleus and Edinger-Westphal nucleus (EW)] are labeled by late stages of infection. Infection of the EW precedes infection in retinorecipient structures, raising the possibility that the SCN becomes infected by retrograde transsynaptic infection via autonomic (i.e., EW) circuits. We tested this hypothesis in two ways: (1) by removing the infected eye $24 \mathrm{hr}$ after PRV 152 inoculation, well before viral infection first appears in the SCN; and (2) by examining central infection after intravitreal PRV 152 injection in animals with ablation of the EW. The pattern and time course of central infection were unchanged after enucleation, whereas EW ablation before intravitreal inoculation eliminated viral infection in the SCN. The results of EW lesions along with known connections between EW, OPN, and SCN indicate that intravitreal injection of PRV Bartha produces a retrograde infection of the autonomic innervation of the eye, which subsequently labels a restricted set of retinorecipient nuclei via retrograde trans-synaptic infection. These results, taken together with other genetic data, indicate that the mutations in PRV Bartha render the virus incapable of anterograde transport. PRV Bartha is thus a retrograde transsynaptic marker in the CNS.

Key words: pseudorabies virus; PRV Bartha; suprachiasmatic nucleus; trans-synaptic transport; olivary pretectal nucleus; Edinger-Westphal nucleus
Over the past decade, transneuronal tracing with neurotropic viruses has greatly advanced our understanding of multisynaptic circuits in the nervous system (Card, 1998; Loewy, 1998; Enquist et al., 1999). Two strains of pseudorabies virus (PRV Becker and PRV Bartha) have been used with great success to transsynaptically infect CNS structures after peripheral application or direct injection into brain parenchyma in several species (Card et al., 1990, 1991, 1998; Strack and Loewy, 1990; Levine et al., 1994; Jansen et al., 1995; Jasmin et al., 1997; Marson, 1997; O’Donnell et al., 1997; Perez Fontan and Velloff, 1997; Banfield et al., 1998; Larsen et al., 1998; Provencio et al., 1998; Buijs et al., 1999, 2001; Chen et al., 1999; Daniels et al., 1999; Ueyama et al., 1999; Billig et al., 2000; la Fleur et al., 2000; Smith et al., 2000; Valentino et al., 2000; Aston-Jones et al., 2001). PRV Becker is a highly virulent wild-type laboratory strain of PRV, whereas PRV Bartha is an attenuated live vaccine strain of PRV. PRV Bartha is attenuated in part because of a large deletion in the unique short

Received Sept. 28, 2001; revised Dec. 13, 2001; accepted Dec. 19, 2001

This work was supported by National Institutes of Health Grants NS 35366, NS 35615, MH 62296 (G.E.P.), and NS 133506 (L.E.W.). We thank Dr. Malcolm Ogilvie for assistance.

Correspondence should be addressed to Dr. Gary E. Pickard, Department of Anatomy and Neurobiology, Colorado State University, Fort Collins, CO 805231670. E-mail: gpickard@lamar.colostate.edu.

Copyright (C) 2002 Society for Neuroscience $\quad 0270-6474 / 02 / 222701-10 \$ 15.00 / 0$ region of the viral genome that eliminates three genes encoding membrane proteins (Lomniczi et al., 1987).

PRV Becker and PRV Bartha transsynaptically label neurons in the rodent brain after injection into the vitreous chamber of the eye, albeit with markedly different patterns of infection (Card et al., 1991; Provencio et al., 1998). PRV Becker infects retinal ganglion cells and is transported anterogradely via the optic nerve, resulting in transsynaptic infection of central visual structures, including the dorsal lateral geniculate nucleus (dLGN), superior colliculus (SC), and suprachiasmatic nucleus (SCN) (Card et al., 1991; Provencio et al., 1998). Intravitreal injection of PRV Bartha produces a central infection in a very restricted set of retinorecipient structures, labeling only the $\mathrm{SCN}$, intergeniculate leaflet (IGL), olivary pretectal nucleus (OPN), and lateral terminal nucleus (LTN) (Card et al., 1991; Levine et al., 1994; Moore et al., 1995; Provencio et al., 1998). The limited infection in retinorecipient structures after intravitreal application of PRV Bartha has been interpreted as a restricted tropism of PRV Bartha for a subset of retinal ganglion cells, thus resulting in infection in a restricted subset of retinorecipient structures after anterograde transport (Card et al., 1991). Further work by Enquist et al. (1994) called this interpretation into question. In addition, recent genetic analysis indicated that PRV Bartha might be incapable of anterograde spread through chains of connected 
neurons (Brideau et al., 2000; Husak et al., 2000; Tomishima and Enquist, 2001). The restricted infection of the SCN and IGL by PRV Bartha apparently by anterograde infection remained enigmatic.

An explanation for this enigma was provided as we examined the pattern and temporal sequence of central infection in the golden hamster after intravitreal injection of recombinants of PRV Becker and PRV Bartha constructed to express the reporter, enhanced green fluorescent protein (EGFP), as a preliminary step in the characterization of retinal ganglion cells afferent to the SCN (Pickard, 1982, 1985; Moore et al., 1995; Smith et al., 2000). Although the patterns of infection observed in the hamster were remarkably similar to those reported in other rodents, further investigation revealed that the infection of retinorecipient structures such as the SCN and IGL after intravitreal PRV Bartha injection results from retrograde transsynaptic transport via autonomic afferents to the eye and not by anterograde transport via the optic nerve.

\section{MATERIALS AND METHODS}

Animals. Syrian hamsters [Mesocricetus auratus, Lak:LVG (Syr), male; Charles River, Kingston, NY] were used in this study. Animals were housed in groups of four per cage and were maintained under a 14/10 hr light/dark cycle with food and water available ad libitum. Animals were received from the supplier at 6-12 weeks of age and were typically not treated with PRV until they had reached an age of 20 weeks. It was found that the time course of infection was less predictable in animals younger than 20 weeks of age, resulting in more fatalities at earlier postinoculation times. Similar findings have been reported for pigs, the natural host of the virus (Smith, 1997).

Recombinant viruses. Recombinant strains of PRV constructed to express a reporter protein were used in this study. PRV 151 is isogenic with PRV Becker, and PRV 152 is isogenic with PRV Bartha. These EGFPexpressing strains were constructed by homologous recombination between a plasmid containing an EGFP expression cassette cloned into the middle of the PRV gG gene and the PRV genome as described previously (Smith et al., 2000; Demmin et al., 2001). Viruses were grown in pig kidney (PK15) cells and stored at $-80^{\circ} \mathrm{C}$. The final titers determined in PK15 cells were $\sim 4 \times 10^{8}$ pfu for PRV 151 and $1 \times 10^{8}$ pfu for PRV 152.

Intraocular injection of virus. Under deep sodium pentobarbital anesthesia $(80 \mathrm{mg} / \mathrm{kg}$, i.p.), animals received a unilateral intravitreal injection of between 1 and $2 \mu \mathrm{l}$ of PRV over a $1 \mathrm{~min}$ interval by using a $10 \mu \mathrm{l}$ Hamilton (Reno, NV) syringe fitted with a 26 gauge needle; the needle was left in place an additional 4 min before removing it from the eye. A fresh stock of virus was thawed for each injection. Animals were maintained in a biosafety level 2 laboratory for up to $122 \mathrm{hr}$ after injection. The Colorado State University Animal Care and Use Committee approved all procedures used in this study.

Tissue preparation. Animals were deeply anesthetized with sodium pentobarbital (100 mg/kg, i.p.) and perfused transcardially with $0.9 \%$ saline followed by freshly prepared fixative consisting of $4 \%$ paraformaldehyde in phosphate buffer $(0.1 \mathrm{M}), \mathrm{pH} 7.3$. Brains were removed, stored in the same fixative containing $30 \%$ sucrose at $4^{\circ} \mathrm{C}$ overnight, and sectioned at $40 \mu \mathrm{m}$ in the coronal plane on a sliding microtome equipped with a freezing stage (Physitemp Instruments Inc., Clifton, NJ). Sections were collected in phosphate buffer, mounted on subbed slides, blotted to remove excess buffer, and coverslipped with Vectashield mounting medium (Vector Laboratories, Burlingame, CA). Coverslips were sealed with fingernail polish to prevent dehydration, and slides were stored in the dark at $4^{\circ} \mathrm{C}$. EGFP fluorescence was stable under these conditions for several months with minimal quenching. Slides were examined using a Leica (Nussloch, Germany) DMRA light microscope equipped with epifluorescence and fitted with a microstepping servomotor in the $z$-axis. Images of EGFP-labeled cells were captured using a Hamamatsu (Hamamatsu City, Japan) C4742-95 CCD digital camera under epifluorescence using EGFP optics (41020 High Q narrow band EGFP filter; Chroma, Brattleboro, VT) and deconvolved using Openlab fluorescence deconvolution software (Improvision, Boston, MA) running on an Apple Macintosh G-4 platform. Digital images were pseudo-colored, and montages of images were prepared using Adobe Photoshop version 5.5. Images were enhanced for brightness and contrast. Eyes were dissected out after fixation, and the retinas were prepared as whole mounts as described previously (Pickard, 1982) and viewed under EGFP optics.

Edinger-Westphal nucleus lesions, superior cervical ganglionectomy, and orbital enucleation. Animals were deeply anesthetized with sodium pentobarbital $(80 \mathrm{mg} / \mathrm{kg}$, i.p.) and positioned in a Kopf stereotaxic apparatus for Edinger-Westphal nucleus (EW) lesioning. Using coordinates determined empirically in test animals, radio frequency lesions aimed at the EW along the midline were made using a Radionics lesion maker (RFG4-A; $80^{\circ} \mathrm{C}$ for $60 \mathrm{sec}$ ). After lesions were made, the craniotomy was filled with Gelfoam (Upjohn, Kalamazoo, MI), and the incision was sutured.

Unilateral superior cervical ganglionectomy (SCG-X) was performed by Zivic-Miller Laboratories, Inc. (Allison Park, PA) on hamsters reared by Charles River. SCG-X animals displayed ptosis in the eye ipsilateral to the ganglionectomy; completeness of the ganglionectomy was further verified by examining the thoracic spinal cord for labeled preganglionic neurons after intravitreal injection of PRV 152 into the ipsilateral eye.

Three animals were enucleated $24 \mathrm{hr}$ after intravitreal PRV 152 injection. Animals were deeply anesthetized with sodium pentobarbital $(80 \mathrm{mg} / \mathrm{kg}$, i.p.); the inoculated eye was quickly removed; the orbit was packed with Gelfoam; and the lids were sutured.

\section{RESULTS}

Intravitreal injection of the PRV Bartha recombinant PRV 152 resulted in a highly reproducible pattern of viral infection in the hamster brain. Moreover, the temporal sequence of infected structures was very reproducible from animal to animal (e.g., EW infection always preceded infection in the SCN), although the extent of central infection as a function of postinjection survival was more variable than has been reported for rats and mice and may be a consequence of the differences in susceptibility to PRV infection in outbred hamsters (Levatte et al., 1998).

\section{Temporal sequence of central infection after intravitreal PRV 152 injection}

By $48 \mathrm{hr}$ after injection, EGFP-labeled cells were observed in the midbrain EW and the intermediolateral nucleus (IML) of the thoracic spinal cord. At progressively later postinjection survival times, the extent of central infection increased. Between 48 and $72 \mathrm{hr}$ after injection, numerous PRV 152-labeled neurons were noted in the EW-oculomotor nuclear complex. At this relatively early stage of infection, labeled EW neurons were observed along the midline, almost exclusively ipsilateral to the injected eye (Fig. $1 a$ ); labeled oculomotor neurons, which tended to be larger than the labeled EW neurons, were located more laterally in the EW-oculomotor complex (Fig. 1a). By this stage of infection, the EGFP in infected neurons in the EW complex had diff used from the labeled cell bodies into dendrites and axons (Fig. 1a), making it possible to trace the labeled axons from the EW complex into the oculomotor nerve as it exited the base of the midbrain. The hypothalamic paraventricular nucleus (PVN; Fig. 1c) and the OPN (Fig. 1d) were also labeled between 48 and $72 \mathrm{hr}$ after injection. The PVN was labeled primarily in its caudal aspect, and the number of infected cells in the PVN ipsilateral to the injected eye was always much greater than the number of labeled cells in this structure contralateral to the injected eye (Fig. 1c). The OPN was labeled bilaterally with a slightly stronger infection contralateral to the injected eye (data not shown). At this early stage of infection, labeled neurons were also first noted in the region of the hypothalamic suprachiasmatic nucleus. However, the infected cells were not located within the SCN, but rather, they were located bilaterally on the dorsolateral border of the SCN (Fig. 1b).

At the midstage of infection, $\sim 72-96 \mathrm{hr}$ after injection, more labeled neurons were observed in the EW, PVN, and OPN relative to the early stage infection. The nucleus of the posterior 

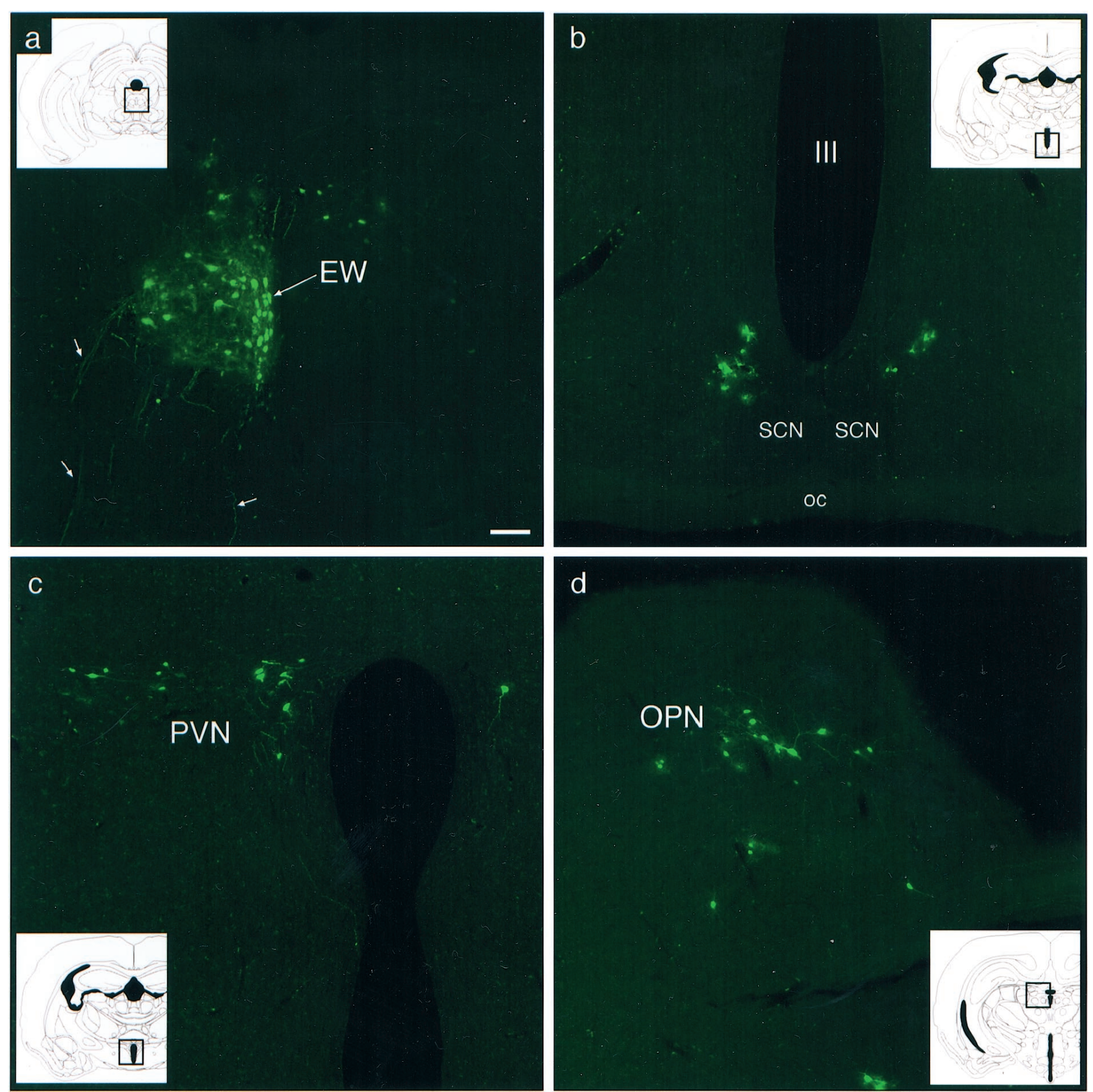

Figure 1. PRV 152-infected nuclei at an early stage of infection (60 hr after intravitreal injection). $a$, Example of transsynaptically infected neurons in the midbrain EW. EW preganglionic parasympathetic neurons are located along the midline ipsilateral to the injected eye. Labeled cells located more laterally may be motor neurons. EGFP-filled axons (short arrows) are seen descending from the EW complex and can be traced into the oculomotor nerve as it emerges from the base of the midbrain. $b$, Early stage pattern of bilateral labeling in the region of the hypothalamic SCN. The first labeled neurons in this region are found in the peri-SCN area, on the dorsolateral boundary of the SCN. Only an occasional labeled cell is found in the SCN at the early stage of infection. $c$, Early stage pattern of bilateral infection in the caudal aspect of the hypothalamic PVN. The majority of labeled neurons are located in the PVN ipsilateral to the injected eye. $d$, Early stage pattern of infection in the OPN. The OPN is labeled bilaterally, although only the OPN ipsilateral to the injected eye is illustrated. Insets illustrate the level of the neural axis from which the photomicrographs were generated; boxed areas depict the approximate boundaries of $a-d$. Coronal hamster brain sections in the insets are from Morin and Wood (2001) with slight modifications. Magnification is the same in $a-d$. Scale bar, $100 \mu \mathrm{m}$. III, Third ventricle; $O C$, optic chiasm.

commissure (NPC) was also consistently labeled at this midstage of infection (data not shown). At this time, labeled cells were also observed scattered throughout the rostrocaudal extent of the SCN (Fig. 2a). The intergeniculate leaflet and the lateral terminal nucleus of the accessory optic system also contained PRV 152labeled neurons beginning between 72 and $96 \mathrm{hr}$ after injection.
By the late stage of infection, $\sim 96-120 \mathrm{hr}$ after injection, the entire SCN was labeled bilaterally throughout its entire extent (Fig. 2b). The OPN (Fig. 2c) and the LTN (Fig. 2e) were also heavily infected bilaterally. At this stage of infection, many more labeled cells were also observed in the caudal PVN (Fig. 2d). Labeled cells in the PVN were noted primarily in the dorsal 

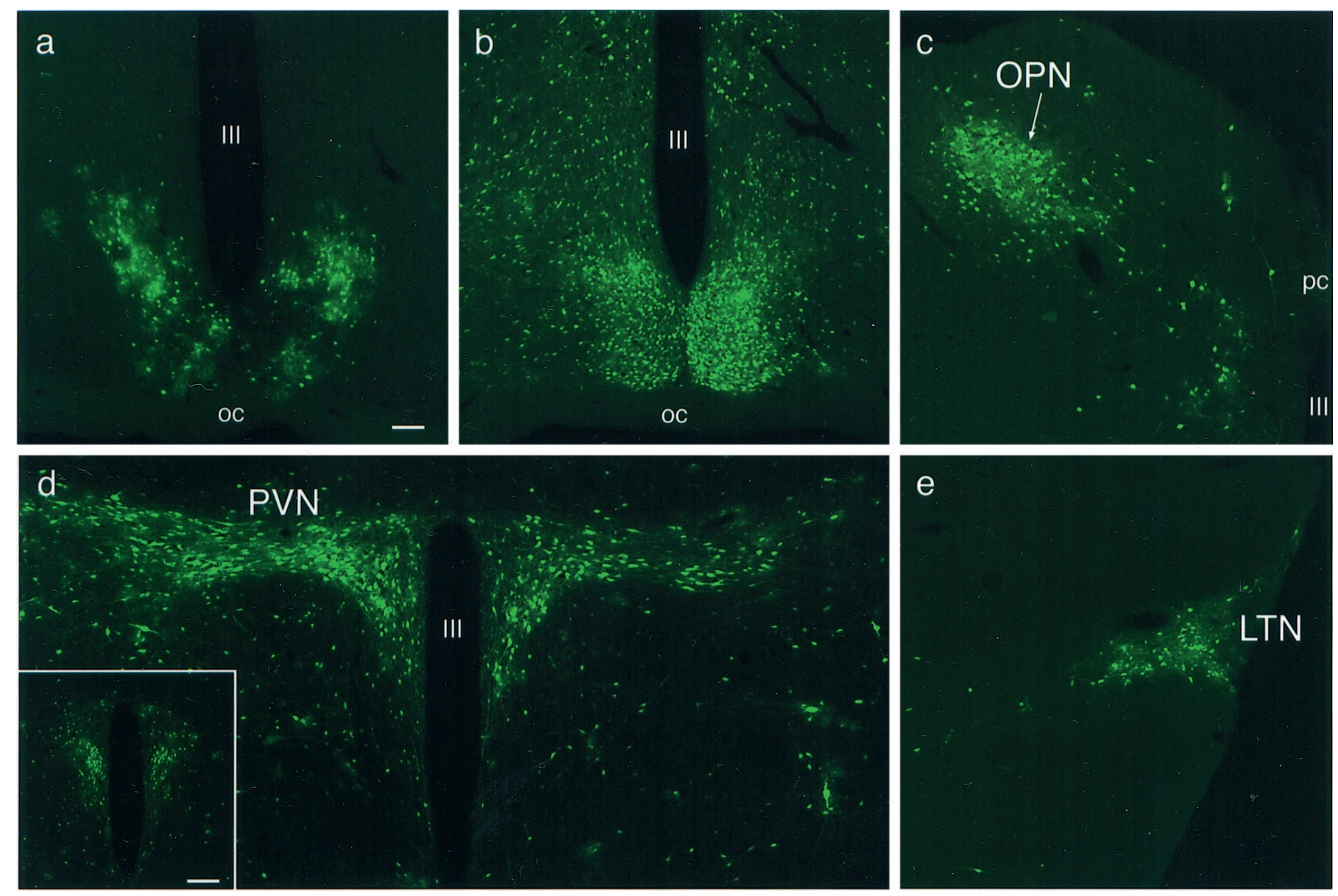

Figure 2. PRV 152-infected neurons at mid ( $72-96 \mathrm{hr})$ and late $(96-120 \mathrm{hr})$ stages of infection after intravitreal injection. $a$, By $72-96 \mathrm{hr}$ after injection, labeled neurons are observed scattered throughout the rostrocaudal aspects of the entire SCN; the infection in the caudal SCN is illustrated. At the late stage of infection (i.e., $120 \mathrm{hr}$ after injection), the entire SCN is labeled bilaterally $(b)$. The OPN $(c)$, PVN $(d)$, and LTN of the accessory optic system $(e)$ are also heavily infected bilaterally at $120 \mathrm{hr}$ after injection, although only the ipsilateral OPN $(c)$ and contralateral LTN (e) are shown. The inset in $d$ illustrates bilateral infection at $120 \mathrm{hr}$ after injection in the rostral aspects of the PVN. Magnification is the same in $a-e$. Scale bars: $a, 100 \mu \mathrm{m} ; d$, inset, $200 \mu \mathrm{m}$. III, Third ventricle; $O C$, optic chiasm.

parvocellular subdivision, the lateral parvocellular subdivision, and the ventral part of the dorsomedial parvocellular subdivision bilaterally (for a description of PVN subdivisions, Morin and Blanchard, 1993; Hallbeck et al., 2001). The more rostral aspects of the PVN were also infected bilaterally at this stage of infection (Fig. $2 d$, inset). The IGL was also heavily infected bilaterally at the late stage of infection (see Fig. $4 a$ ).

\section{Labeled retinal ganglion cells}

At the late stage of infection, the retina contralateral to the injected eye contained numerous EGFP-expressing ganglion cells. Because EGFP in the PRV 152-infected ganglion cells diffuses throughout the entire neuron, the complete dendritic arbor of labeled cells could be visualized in retinal whole mounts without further tissue processing. Retinal ganglion cells expressing EGFP displayed several different morphologies, as illustrated in Figure 3. The multiple morphological types of labeled ganglion cells observed in the contralateral retina were expected, because four retinorecipient nuclei with diverse functions contributed to the retrograde labeling of ganglion cells in the retina. Studies are currently under way to describe the complete dendritic morphology of ganglion cells afferent to the SCN.

\section{Enucleation of the PRV 152-infected eye $24 \mathrm{hr}$ after infection}

Although the SCN, IGL, OPN, and LTN are all retinorecipient nuclei and thus might become infected with PRV 152 by anterograde transport via the optic nerve after intravitreal injection, the temporal sequence of infection (i.e., EW labeled before SCN and IGL) raised the possibility that the retinorecipient structures could also become labeled via retrograde transsynaptic transport of PRV as a consequence of infection of the autonomic afferents to the eye. We reasoned, therefore, that if PRV were reaching the SCN and IGL via the optic nerve, removal of the inoculated eye $24 \mathrm{hr}$ after infection would eliminate the PRV 152 label in the SCN and IGL as a result of cutting the optic nerve before the beginning of anterograde transport of the virus. (Infected neurons in the hamster peri-SCN region are typically not observed before $\sim 60 \mathrm{hr}$ after intravitreal injection, and labeled neurons in the IGL are first noted $\sim 72 \mathrm{hr}$ after injection.) Enucleation of the infected eye $24 \mathrm{hr}$ after injection $(n=3)$ had no effect on the pattern of label observed in the SCN and IGL or in any of the other retinorecipient nuclei when the animals were examined $120 \mathrm{hr}$ after infection (Fig. 4a,b). This finding suggested that PRV 152 may not be infecting retinorecipient structures by antero- 


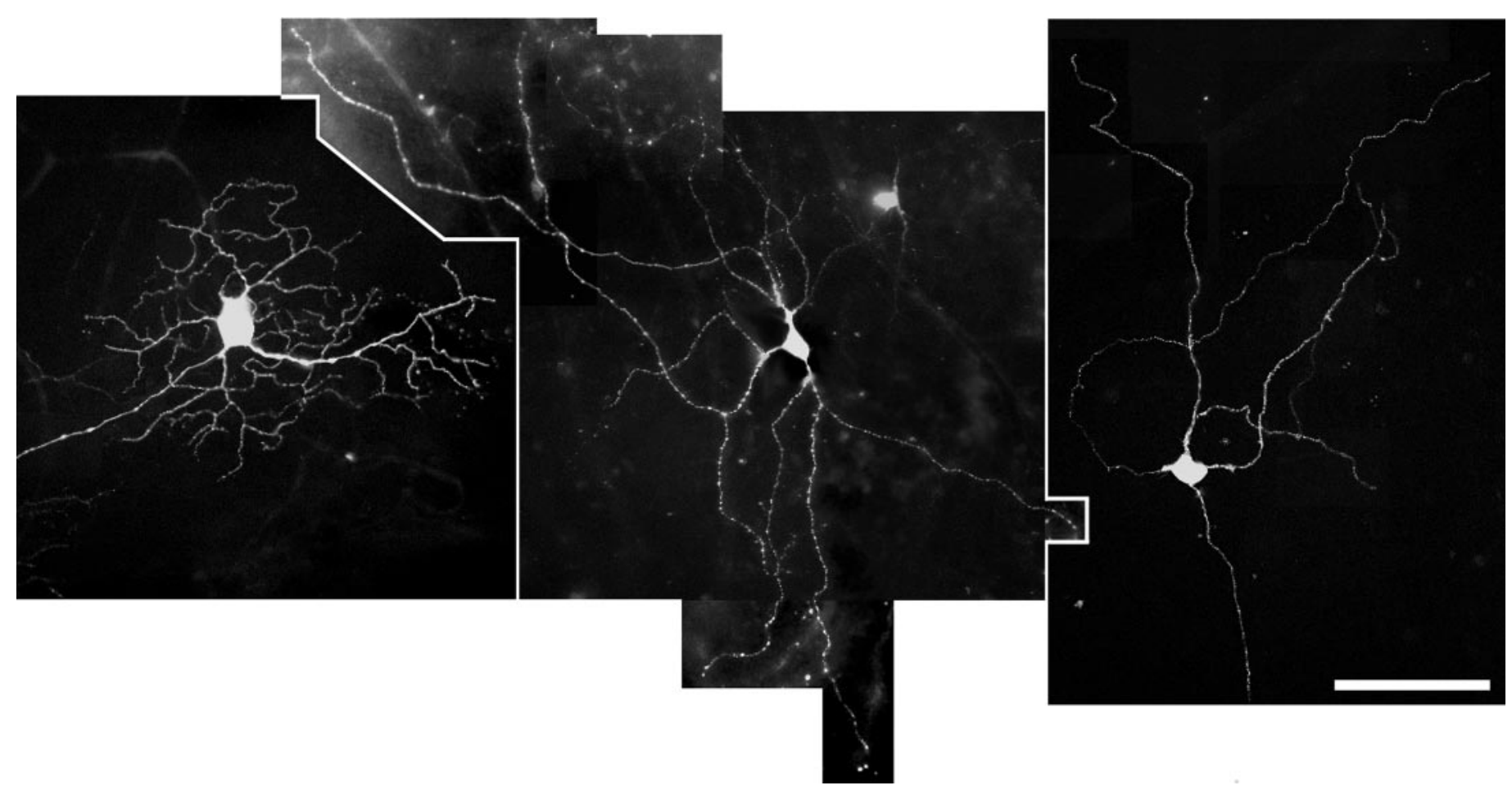

Figure 3. PRV 152-labeled retinal ganglion cells in the retina contralateral to the injected eye at $120 \mathrm{hr}$ after injection. EGFP has diff used throughout the entire dendritic arbor of these retrogradely transsynaptically infected ganglion cells viewed in a retinal whole mount. The several different morphological types of retinal ganglion cells that are apparent may have been infected after retrograde transport from the SCN, IGL, OPN, or LTN, because all four retinorecipient structures are labeled. Scale bar, $100 \mu \mathrm{m}$.

grade transport through the optic nerve; therefore, another route by which PRV 152 could infect retinorecipient structures by retrograde transport was examined.

\section{Edinger-Westphal nucleus lesions}

One set of autonomic afferents to the eye arises from parasympathetic preganglionic neurons in the EW nuclear complex of the midbrain. EW neurons send preganglionic axons through the oculomotor nerve to innervate postganglionic neurons in the ciliary ganglion. Postganglionic neurons in the ciliary ganglion innervate smooth muscles in the iris and ciliary body that mediate pupillary constriction and lens accommodation, respectively. Because EW neurons were labeled by the early stage of infection, we hypothesized that the EW may provide a route by which retinorecipient nuclei become infected via retrograde transport.

To evaluate the potential contribution of the EW-ciliary ganglion parasympathetic circuit to the eye, lesions were made in the midbrain aimed at the EW in 18 animals. At least 1 week after recovering from the surgery, animals received unilateral intravitreal injections of PRV 152 as in intact animals and were killed either $96 \mathrm{hr}$ after injection $(n=2)$ or $114-122 \mathrm{hr}$ after injection $(n=16)$ to ensure sufficient time for robust transsynaptic labeling in the brain. In animals with midbrain lesions that ablated almost the entire EW complex $(n=6$; Fig. 5), the SCN and IGL were virtually unlabeled (Fig. $4 d-f$ ). Although the SCN was virtually devoid of infected neurons, the anterior hypothalamus surrounding the SCN remained heavily labeled (Fig. $4 d-f$ ). Labeling in the OPN and LTN was greatly reduced in these cases, although not eliminated completely (data not shown). In animals with less than complete EW destruction that tended to spare the more caudal aspects of the nucleus $(n=4)$, labeling in the OPT, IGL, and
SCN was diminished in relative proportion to the completeness of EW ablation (data not shown). Lesions that spared the EW complex completely $(n=8)$ were typically placed rostral and somewhat dorsal to the EW. These lesions reduced labeling very little in the SCN, IGL, OPN, and LTN (Fig. 4c).

\section{Superior cervical ganglionectomy}

The other set of autonomic afferents to the eye arises from preganglionic sympathetic neurons in the IML of the thoracic spinal cord. These neurons innervate postganglionic neurons in the SCG. SCG cells send postganglionic axons to innervate smooth muscles in the iris responsible for pupillary dilation. In this study, the IML became infected at approximately the same time as the EW. The sympathetic afferents to the eye arising in the thoracic spinal cord and SCG have been labeled in the rat after PRV Bartha injection into the anterior chamber of the eye (Strack and Loewy, 1990). To evaluate the potential contribution of sympathetic afferents to the eye to the pattern of infection revealed after intravitreal injection of PRV 152, unilateral SCG-X was performed $(n=12)$, and the animals were allowed to recover for several weeks. Intravitreal PRV 152 injections were made in the eye ipsilateral to the removed SCG, and the animals were examined at $24 \mathrm{hr}$ intervals after injection up to $120 \mathrm{hr}$ after injection. Unlike SCG-intact animals, where label was observed in the IML, SCG-X eliminated label in the spinal cord (data not shown) but had little other impact on the pattern of infection except for a reduction in the label noted in the hypothalamic PVN. The autonomic circuits from the eye to retinorecipient structures in the brain are well characterized (see Discussion) and are summarized in Figure 6. 

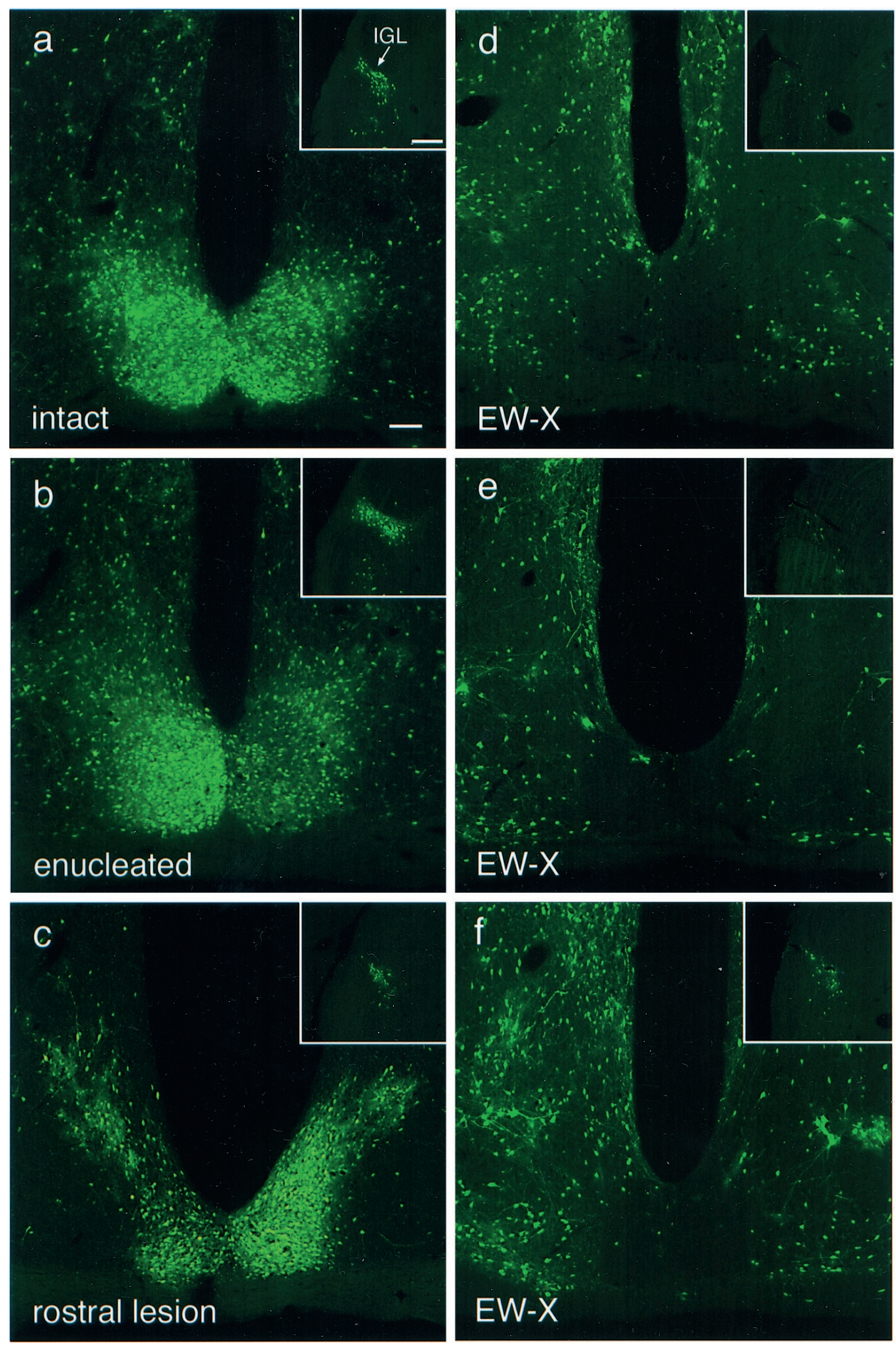

Figure 4. PRV 152-labeled neurons in the SCN and IGL $120 \mathrm{hr}$ after intravitreal injection in intact and lesioned animals. $a$, Pattern of bilateral infection typically observed in the caudal SCN and IGL (inset) in a neurologically intact animal. $b$, Pattern of infection in the caudal SCN and IGL (inset) in an animal in which the infected eye was removed $24 \mathrm{hr}$ after injection. The pattern of infection in the SCN and IGL is similar to the pattern in the SCN and IGL in the intact animal illustrated in $a . c$, Example of the pattern of infection in the SCN and IGL (inset) in an animal in which the midbrain lesion was placed rostral to the EW, sparing almost the entire nucleus. Note that the robust labeling of the SCN is comparable with (Figure legend continues.) 


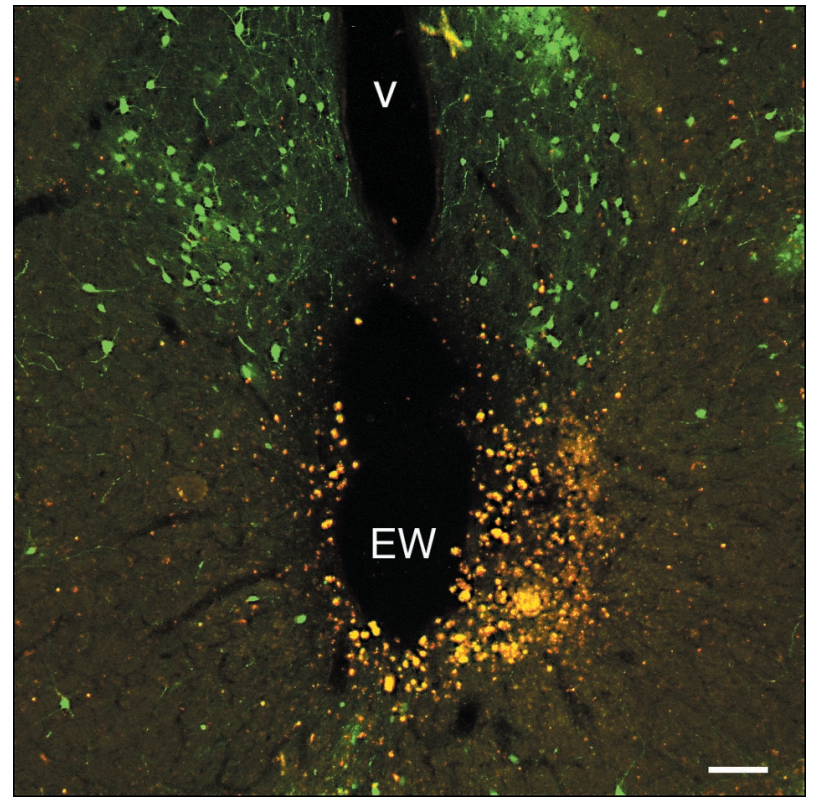

Figure 5. Midbrain lesion in which the EW was destroyed. An example of a midbrain EW lesion in an animal in which label in the SCN and IGL was eliminated (SCN and IGL labeling in this animal are depicted in Fig. $4 d)$. The lesion shown is in the midbrain at the level of the oculomotor nerve root that destroyed the EW bilaterally (compare with Fig. 1a). The lesion extended approximately another $600 \mu \mathrm{m}$ caudal from the level illustrated. Autofluorescence from damaged neurons and red blood cells appears as yellow-orange fluorescence. EGFP-labeled neurons in the periaqueductal gray dorsal to the EW are evident. $V$, Ventricle. Scale bar, $100 \mu \mathrm{m}$.

\section{PRV 151 intravitreal injections}

To confirm the labeling of "visual structures" (i.e., dLGN and SC) in the hamster after intravitreal injection of a wild-type PRV, injections were made using PRV 151, a recombinant strain of PRV Becker constructed to express EGFP. Animals inoculated with PRV 151 demonstrated symptoms of discomfort $\sim 50-72 \mathrm{hr}$ after injection $(n=6)$. Three of six animals examined between 50 and $72 \mathrm{hr}$ after injection revealed localized infection in the dLGN and the SC (Fig. 7a,b), a pattern of labeling never observed in animals infected with the PRV Bartha recombinant strain PRV 152. The focal infection in these visual structures represented an early stage of anterograde infection (Card et al., 1991). In the three remaining PRV 151-injected animals, no infected neurons were observed in any visual structures, whereas infection in the EW was clearly evident in each case. Apparently these animals were killed at an even earlier stage of anterograde infection (compared with the three animals with infection in the dLGN and $\mathrm{SC}$ ), although they displayed severe behavioral symptoms.

\section{DISCUSSION}

The present study conducted in the golden hamster demonstrates that intravitreal injection of recombinant strains of PRV expressing the EGFP reporter results in a pattern of central infection

\section{Sympathetic}

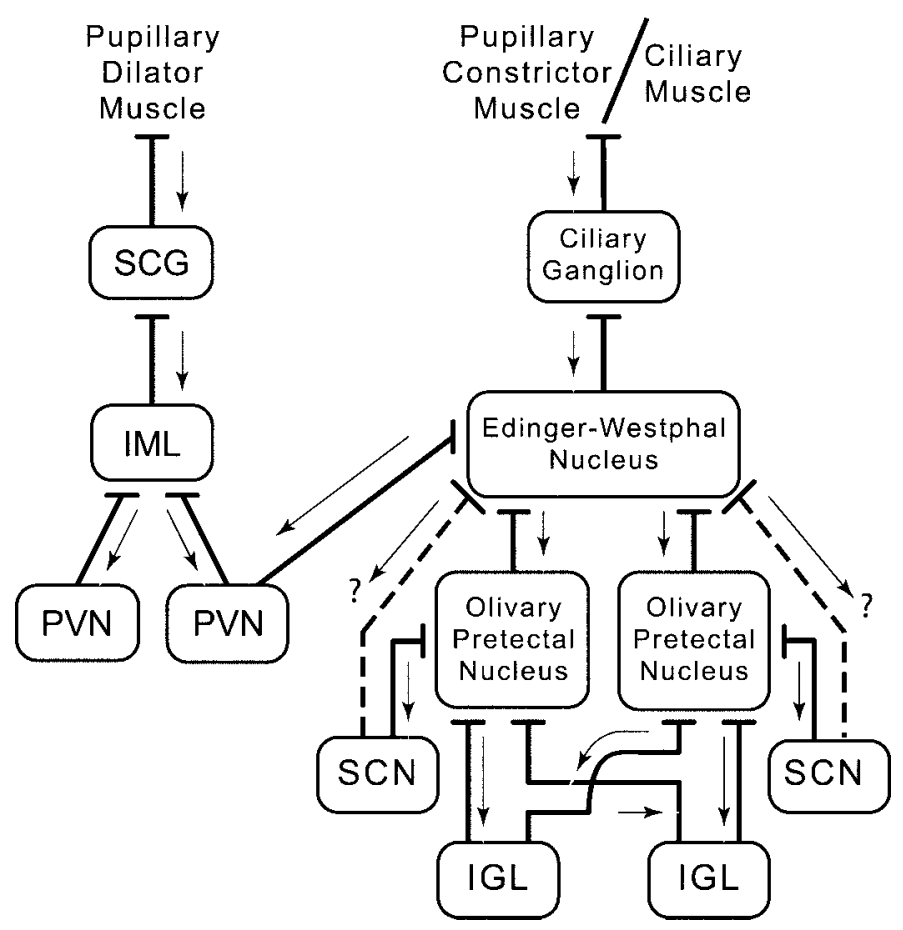

Figure 6. Diagram summarizing the sympathetic and parasympathetic innervation of the smooth muscle of the eye. Sympathetic afferents arising from postganglionic neurons in the SCG innervate smooth muscle in the iris and mediate pupillary dilation. The IML in the thoracic spinal cord sends preganglionic efferents to the SCG, and the IML is innervated bilaterally by long descending projections from the hypothalamic PVN. Parasympathetic afferents arising from postganglionic neurons in the ciliary ganglion innervate smooth muscle in the iris and ciliary body of the eye that mediate pupillary constriction and lens accommodation, respectively. The EW of the midbrain sends preganglionic efferents to the ciliary ganglion. EW receives afferents from the OPN bilaterally and also perhaps (?) from the SCN. The OPN receives afferents from the IGL bilaterally. The LTN (diagram not shown) is afferent to the OPN, IGL, or both. Arrows indicate the direction of retrograde transsynaptic transport of PRV 152 (from postsynaptic neuron to presynaptic neuron) after intravitreal injection.

similar to that described previously in the rat (Card et al., 1991; Whealy et al., 1993; Levine et al., 1994; Moore et al., 1995) and the mouse (Provencio et al., 1998) after intravitreal PRV Bartha and PRV Becker injections. Intravitreal PRV Bartha injection labels a restricted subset of nuclei in the rodent brain known to receive direct afferents from the retina. This result led to the suggestion that PRV Bartha has a selective affinity for a specific subset of retinal ganglion cells (Card et al., 1991), a suggestion that was called into question by the complementation analyses of Enquist et al. (1994). The results of the present study provide an explanation for the apparently paradoxical infections of retinorecipient nuclei by PRV Bartha. We suggest that all central labeling by PRV Bartha after intravitreal injection results from retrograde spread through autonomic routes.

\footnotetext{
(Figure legend continued.) that in an intact animal $(a)$. The slight reduction in label in the IGL may be attributable to disruption of fibers of passage from the IGL to OPN. $d-f$, Examples of the pattern of infection in the SCN and IGL (insets) in animals in which the midbrain lesions destroyed almost the entire EW (Fig. 5), sparing, if any, only the most rostral aspect of the EW. The SCN and IGL are virtually devoid of infected neurons in $d$ and $e$. The midbrain lesion in the animal illustrated in $f$ eliminated infection in the SCN, whereas infection in the IGL was greatly reduced compared with that in intact animals $(a)$. The SCN ipsilateral to the injected eye is on the left in all images. The ipsilateral IGL is shown in all images. $E W$ - $X$, Midbrain lesion that ablated the EW. Magnification is the same in $a-f$. Scale bars: $a, 100 \mu \mathrm{m} ; a$, inset, $200 \mu \mathrm{m}$.
} 

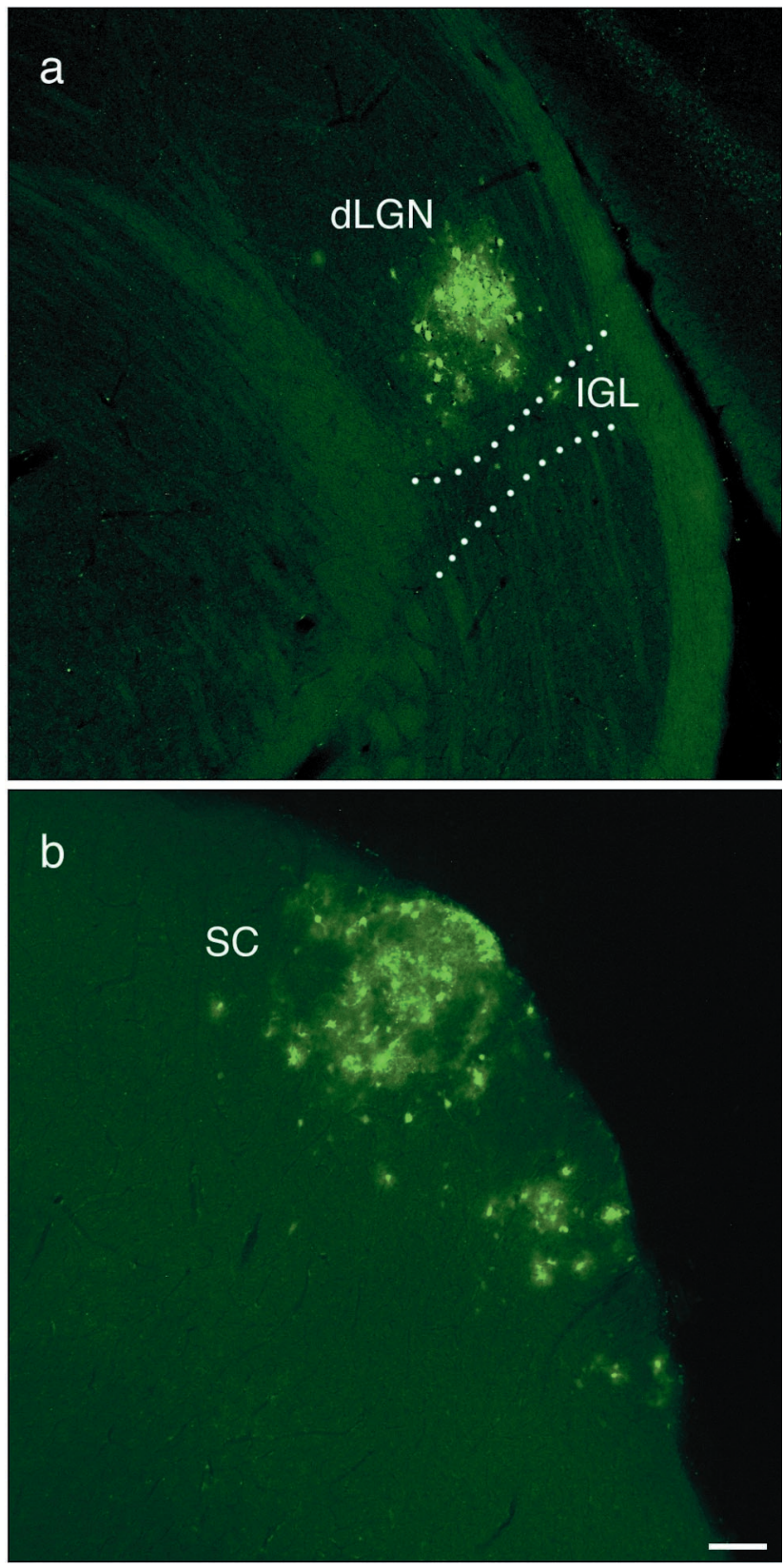

Figure 7. Labeled neurons in the dLGN and SC after intravitreal injection of PRV 151. Localized patches of neurons in the dLGN and SC are transsynaptically infected $52 \mathrm{hr}$ after injection after anterograde transport of virus via the optic nerve. Scale bar, $100 \mu \mathrm{m}$.

If PRV Bartha injected into the vitreous was transported anterogradely via the optic nerve to transsynaptically infect the $\mathrm{SCN}$, midbrain lesions that destroy the EW would have had a negligible effect on that infection. However, EW ablation before intravitreal injection of PRV 152 completely eliminated PRV labeling in the SCN and eliminated or greatly reduced labeling in other retinorecipient nuclei. Moreover, removal of an eye infected with PRV 152 did not eliminate transsynaptic infection in the SCN, a finding inconsistent with virus being transported anterogradely via the optic nerve. Instead, these findings are consistent with a route of infection of the select subset of retinorecipient structures in the brain (e.g., the SCN) by retrograde transsynaptic infection of autonomic circuits rather than anterograde infection via visual circuits.

\section{Autonomic circuits afferent to the eye}

The temporal sequence of PRV 152 infection (i.e., EW infected before retinorecipient nuclei), coupled with the well documented neuroanatomical and functional connections between the labeled structures, alerted us to the possibility that the pattern of infection revealed after intravitreal PRV 152 injection may be the result of retrograde transport via autonomic afferents to the eye. The pathways involved in the parasympathetic regulation of the pupillary light reflex are very well known (Ranson and Magoun, 1933). Retinal ganglion cells sensitive to changes in ambient illumination project to the olivary pretectal nuclei (Scalia, 1972; Young and Lund, 1998) that contains luminance-sensitive neurons (Clarke and Ikeda, 1985a,b; Gamlin et al., 1995). The OPN projects bilaterally to the Edinger-Westphal nucleus (Itaya and Van Hoesen, 1982; Campbell and Lieberman, 1985; Young and Lund, 1994; Klooster et al., 1995), the preganglionic parasympathetic nucleus mediating light-induced pupillary constriction. EW sends efferents via the oculomotor nerve to the ipsilateral ciliary ganglion, which in turn sends postganglionic parasympathetic axons to the constrictor muscles of the iris, resulting in pupilloconstriction (Oyster, 1999). In addition, efferents from the ciliary ganglion also innervate the ciliary muscle of the eye, which subserves the accommodation reflex (Oyster, 1999).

The OPN may be one of the key structures in the previous misinterpretation of the route by which intravitreal PRV Bartha transsynaptically infects other nuclei innervated by the retina (Fig. 6). As described above, the OPN becomes retrogradely infected bilaterally after the initial infection in the EW ipsilateral to the PRV 152-injected eye. The fact that the OPN becomes infected bilaterally and receives afferents bilaterally from the IGL in the hamster (Morin and Blanchard, 1998) and the rat (Moore et al., 2000) provides an explanation for the bilaterally equal infection observed in the hamster OPN and IGL in this study and in previous reports in other rodents (Card et al., 1991; Moore et al., 1995; Provencio et al., 1998). The LTN could become retrogradely infected by either of two routes, from either the OPN (Blanks et al., 1995) or the IGL (Moore et al., 2000). The NPC is also consistently labeled bilaterally, and it innervates the EW bilaterally (Young and Lund, 1994).

Infection in the SCN may also arise as a retrograde transsynaptic infection from the OPN; Leak and Moore (2001) have shown that the SCN projects to the OPN in the rat. However, the SCN-to-OPN pathway in the hamster may be somewhat less direct than that of the rat. Afferents to the OPN from the SCN in the hamster do not arise from within the SCN proper but rather from the peri-SCN region immediately dorsal to the caudal aspects of the nucleus (Morin and Blanchard, 1998). In the early and mid stages of infection, cells in the peri-SCN region are the first to be infected in the hypothalamus, with the SCN becoming progressively infected with increasing postinjection time. This pattern of infection may indicate that SCN neurons innervate neurons in the peri-SCN area. However, the SCN (or peri-SCN) may also send efferents directly to the EW (Kucera and Favrod, 1979; Gamlin et al., 1982; Watts, 1991). The SCN could also get infected after retrograde transsynaptic transport from the PVN. The PVN is afferent to the EW (Swanson, 1977; Luiten et al., 1985; Holstege, 1987; Zheng et al., 1995), and the SCN is afferent to the PVN region (Watts et al., 1987; Buijs et al., 1993; Kalsbeek et al., 1993; Teclemariam-Mesbah et al., 1997). Midbrain lesions in this study that included the mid through caudal EW eliminated infection in the SCN but did not completely eliminate infection in 
the OPN, IGL, and PVN. This finding suggests a direct SCN-EW pathway in the hamster similar to that described in the pigeon (Gamlin et al., 1982). Moreover, the findings suggest that the SCN does not regulate sympathetic outflow directed toward SCG neurons that regulate pupillary dilation, unlike SCN regulation of SCG neurons that regulate pineal melatonin synthesis (Larsen et al., 1998).

The sympathetic input to the eye is also well understood. Afferents to the iris dilator muscle arise from preganglionic neurons in the IML of the thoracic spinal cord and from postganglionic neurons in the SCG. SCG-X had little impact on the pattern of central infection after intravitreal injection of PRV 152 except for a reduction in labeled PVN neurons (and elimination of label in the IML); the PVN-to-IML circuit is well described (Saper et al., 1976; Luiten et al., 1985). It appears that the infection of retinorecipient nuclei after intravitreal injection arises from the EW parasympathetic circuit.

\section{PRV Bartha is a retrograde transsynaptic marker in the CNS}

The PRV Bartha strain (and PRV 152) carries a deletion in the unique short (Us) region of the genome that removes all of the $\mathrm{gE}$, gI, and Us9 coding sequences and part of the Us2 coding sequence (Mettenleiter et al., 1985; Petrovskis et al., 1986; Lomniczi et al., 1987). Recent work has suggested that the products of these missing genes affect the direction of spread of PRV in the CNS. Intravitreal injection of the wild-type PRV infects retinal ganglion cells and anterogradely spreads to infect visual centers in the brain, including the dLGN and SC (Card et al., 1991; Provencio et al., 1998; this study). In contrast, intravitreal injection of gE-, gI-, and Us9-null mutants does not produce an infection in the dLGN or SC but rather results in the infection of a restricted set of retinorecipient nuclei similar to those infected after PRV Bartha intravitreal injection (Brideau et al., 2000; Husak et al., 2000). It is clear from the present study that the restricted set of retinorecipient nuclei that become infected with PRV Bartha or the null mutants described above are done so by retrograde transneuronal transport via autonomic circuits to the eye.

Similarly, intracerebral injection of the wild-type PRV produces bidirectional (anterograde and retrograde) transport of the virus (Card et al., 1998), whereas PRV Bartha is transported from the site of injection in only a retrograde direction (Jasmin et al., 1997; O’Donnell et al., 1997; Card et al., 1998; Chen et al., 1999; Aston-Jones et al., 2001). The lipid envelope of PRV virions contains at least 16 virally encoded membrane proteins, including $\mathrm{gE}$, gI, and Us9 (Mettenleiter, 1994). It is now clear that the deletion in the Us region of PRV Bartha renders the virus incapable of anterograde transport in the CNS. Deletion of any one of three contiguous genes in the Us9 region, gI, gE, or Us9, blocks spread of virus from the infected presynaptic neuron to the uninfected postsynaptic neuron (Brideau et al., 2000; Husak et al., 2000). Recent work by Tomishima and Enquist (2001) indicates that Us9 plays a role different from that of $\mathrm{gE}$ and $\mathrm{gI}$ in promoting anterograde spread by specifically effecting entry of viral membrane proteins into axons.

\section{Retrograde spread of PRV 152 after intravitreal injection is not specific to the hamster}

PRV Bartha has been reported to be transneuronally transported in a retrograde manner in several species, including the rat, mouse, sheep, ferret, and chicken (Enquist et al., 1999). The golden hamster was used in this study because the hamster has been the species of choice for investigating the functional organization of the SCN and circadian timing system. The interpretation that PRV 152 infects the SCN only via a retrograde route after intravitreal injection is not specific to the hamster. Enucleation of the PRV 152-infected eye in rats and mice $24 \mathrm{hr}$ after intravitreal injection had no effect on the pattern or time course of central infection, similar to the present findings in the hamster (Pickard et al., 2001). Moreover, intravitreal PRV Bartha injection in the hamster produces a pattern of central infection identical to that observed with recombinant PRV 152 (Pickard et al., 1992).

\section{Summary}

The present study demonstrates that intravitreal injection of the PRV Bartha recombinant PRV 152 labels retinorecipient nuclei in the brain such as the SCN by retrograde transport via autonomic afferents to the eye, primarily through the EW parasympathetic circuit. Because the labeling of the SCN after intravitreal PRV Bartha injection was the only system in the CNS in which PRV Bartha had been suggested to travel in an anterograde manner, we suggest that PRV Bartha should be considered a transsynaptic marker that travels exclusively in a retrograde direction in the CNS.

\section{REFERENCES}

Aston-Jones G, Chen S, Zhu Y, Oshinsky ML (2001) A neural circuit for circadian regulation of arousal. Nat Neurosci 4:732-738.

Banfield BW, Yap GS, Knapp AC, Enquist LW (1998) A chicken embryo eye model for the analysis of alphaherpesvirus neuronal spread and virulence. J Virol 72:4580-4588.

Billig I, Foris JM, Enquist LW, Card JP, Yates BJ (2000) Definition of neuronal circuitry controlling the activity of phrenic and abdominal motoneurons in the ferret using recombinant strains of pseudorabies virus. J Neurosci 20:7446-7454.

Blanks RH, Clarke RJ, Lui F, Giolli RA, Van Pham S, Torigoe Y (1995) Projections of the lateral terminal accessory optic nucleus of the common marmoset (Callithrix jacchus). J Comp Neurol 354:511-532.

Brideau AD, Eldridge MG, Enquist LW (2000) Directional transneuronal infection by pseudorabies virus is dependent on an acidic internalization motif in the Us9 cytoplasmic tail. J Virol 74:4549-4561.

Buijs RM, Markman M, Nunes-Cardoso B, Hou Y-X, Shinn S (1993) Projections of the suprachiasmatic nucleus to stress-related areas in the rat hypothalamus: a light and electron microscopic study. J Comp Neurol 335:42-54.

Buijs RM, Wortel J, van Heerikhuize JJ, Feenstra MG, Ter Horst GJ, Romijn HJ, Kalsbeek A (1999) Anatomical and functional demonstration of a multisynaptic suprachiasmatic nucleus adrenal (cortex) pathway. Eur J Neurosci 11:1535-1544.

Buijs RM, Chun SJ, Niijima A, Romijn HJ, Nagai K (2001) Parasympathetic and sympathetic control of the pancreas: a role for the suprachiasmatic nucleus and other hypothalamic centers that are involved in the regulation of food intake. J Comp Neurol 431:405-423.

Campbell G, Lieberman AR (1985) The olivary pretectal nucleus: experimental anatomical studies in the rat. Philos Trans R Soc Lond B Biol Sci 310:573-609.

Card JP (1998) Exploring brain circuitry with neurotropic viruses: new horizons in neuroanatomy. Anat Rec 253:176-185.

Card JP, Rinaman L, Schwaber JS, Miselis RR, Whealy ME, Robbins AK, Enquist LW (1990) Neurotropic properties of pseudorabies virus: uptake and transneuronal passage in the rat central nervous system. J Neurosci 10:1974-1994.

Card JP, Whealy ME, Robbins AK, Moore RY, Enquist LW (1991) Two alpha-herpesvirus strains are transported differentially in the rodent visual system. Neuron 6:957-969.

Card JP, Levitt P, Enquist LW (1998) Different patterns of neuronal infection after intracerebral injection of two strains of pseudorabies virus. J Virol 72:4434-4441.

Chen S, Yang M, Miselis RR, Aston-Jones G (1999) Characterization of transsynaptic tracing with central application of pseudorabies virus. Brain Res 838:171-183.

Clarke RJ, Ikeda H (1985a) Luminance detectors in the olivary pretectal nucleus and their relationship to the pupillary light reflex in the rat. II. Studies using sinusoidal light. Exp Brain Res 59:83-90.

Clarke RJ, Ikeda H (1985b) Luminance and darkness detectors in the olivary and posterior pretectal nuclei and their relationship to the pupillary light reflex in the rat. I. Studies with steady luminance levels. Exp Brain Res 57:224-232. 
Daniels D, Miselis RR, Flanagan-Cato LM (1999) Central neuronal circuit innervating the lordosis-producing muscles defined by transneuronal transport of pseudorabies virus. J Neurosci 19:2823-2833.

Demmin GL, Clase AC, Randall JA, Enquist LW, Banfield BW (2001) Insertions in the $\mathrm{gG}$ gene of pseudorabies virus reduce expression of the upstream Us3 protein and inhibit cell-to-cell spread of virus infection. J Virol 75:10856-10869.

Enquist LW, Dubin J, Whealy ME, Card JP (1994) Complementation analysis of pseudorabies virus $\mathrm{gE}$ and gI mutants in retinal ganglion cell neurotropism. J Virol 68:5275-5279.

Enquist LW, Husak PJ, Banfield BW, Smith GA (1999) Infection and spread of alphaherpesviruses in the nervous system. Adv Virus Res 51:237-347.

Gamlin PD, Reiner A, Karten HJ (1982) Substance P-containing neurons of the avian suprachiasmatic nucleus project directly to the nucleus of Edinger-Westphal. Proc Natl Acad Sci USA 79:3891-3895.

Gamlin PD, Zhang H, Clarke RJ (1995) Luminance neurons in the pretectal olivary nucleus mediate the pupillary light reflex in the rhesus monkey. Exp Brain Res 106:169-176.

Hallbeck M, Larhammar D, Blomqvist A (2001) Neuropeptide expression in rat paraventricular hypothalamic neurons that project to the spinal cord. J Comp Neurol 433:222-238.

Holstege G (1987) Some anatomical observations on the projections from the hypothalamus to brainstem and spinal cord: an HRP and autoradiographic tracing study in the cat. J Comp Neurol 260:98-126.

Husak PJ, Kuo T, Enquist LW (2000) Pseudorabies virus membrane proteins gI and gE facilitate anterograde spread of infection in projection-specific neurons in the rat. J Virol 74:10975-10983.

Itaya SK, Van Hoesen GV (1982) WGA-HRP as a transneuronal marker in the visual pathways of monkey and rat. Brain Res 236:199-204.

Jansen AS, Nguyen XV, Karpitskiy V, Mettenleiter TC, Loewy AD (1995) Central command neurons of the sympathetic nervous system: basis of the fight-or-flight response. Science 270:644-646.

Jasmin L, Burkey AR, Card JP, Basbaum AI (1997) Transneuronal labeling of a nociceptive pathway, the spino-(trigemino-)parabrachioamygdaloid, in the rat. J Neurosci 17:3751-3765.

Kalsbeek A, Teclemariam-Mesbah R, Pevet P (1993) Efferent projections of the suprachiasmatic nucleus in the golden hamster (Mesocricetus auratus). J Comp Neurol 332:293-314.

Klooster J, Vrensen GF, Muller LJ, van der Want JJ (1995) Efferent projections of the olivary pretectal nucleus in the albino rat subserving the pupillary light reflex and related reflexes. A light microscopic tracing study. Brain Res 688:34-46.

Kucera P, Favrod P (1979) Suprachiasmatic nucleus projection to mesencephalic central grey in the woodmouse (Apodemus sylvaticus L.). Neuroscience 4:1705-1715.

la Fleur SE, Kalsbeek A, Wortel J, Buijs RM (2000) Polysynaptic neural pathways between the hypothalamus, including the suprachiasmatic nucleus, and the liver. Brain Res 871:50-56.

Larsen PJ, Enquist LW, Card JP (1998) Characterization of the multisynaptic neuronal control of the rat pineal gland using viral transneuronal tracing. Eur J Neurosci 10:128-145.

Leak RK, Moore RY (2001) Topographic organization of suprachiasmatic nucleus projection neurons. J Comp Neurol 433:312-334.

Levatte MA, Mabon PJ, Weaver LC, Dekaban GA (1998) Simultaneous identification of two populations of sympathetic preganglionic neurons using recombinant herpes simplex virus type 1 expressing different reporter genes. Neuroscience 82:1253-1267.

Levine JD, Zhao X-S, Miselis RR (1994) Direct and indirect retinohypothalamic projections to the supraoptic nucleus in the female albino rat. J Comp Neurol 341:214-224.

Loewy AD (1998) Viruses as transneuronal tracers for defining neural circuits. Neurosci Biobehav Rev 22:679-684.

Lomniczi B, Watanabe S, Ben Porat T, Kaplan AS (1987) Genome location and identification of functions defective in the Bartha vaccine strain of pseudorabies virus. J Virol 61:796-801.

Luiten PGM, ter Horst GJ, Karst H, Steffens AB (1985) The course of paraventricular hypothalamic efferents to autonomic structures in medulla and spinal cord. Brain Res 329:374-378.

Marson L (1997) Identification of central nervous system neurons that innervate the bladder body, bladder base, or external urethral sphincter of female rats: a transneuronal tracing study using pseudorabies virus. J Comp Neurol 389:584-602.

Mettenleiter T (1994) Pseudorabies (Aujeszky's disease) virus: state of the art. Acat Vet Hung 42:153-177.

Mettenleiter TC, Lukacs N, Rziha HJ (1985) Pseudorabies virus avirulent strains fail to express a major glycoprotein. J Virol 56:307-311.

Moore RY, Speh JC, Card JP (1995) The retinohypothalamic tract originates from a distinct subset of retinal ganglion cells. J Comp Neurol 352:351-366.

Moore RY, Weis R, Moga MM (2000) Efferent projections of the intergeniculate leaflet and the ventral lateral geniculate nucleus in the rat. J Comp Neurol 420:398-418.

Morin LP, Blanchard JH (1993) Organization of the hamster paraventricular hypothalamic nucleus. J Comp Neurol 332:341-357.
Morin LP, Blanchard JH (1998) Interconnections among nuclei of the subcortical visual shell: the intergeniculate leaflet is a major constituent of the hamster subcortical visual system. J Comp Neurol 396:288-309.

Morin LP, Wood RI (2001) A stereotaxic atlas of the golden hamster brain. New York: Academic.

O'Donnell P, Lavin A, Enquist LW, Grace AA, Card JP (1997) Interconnected parallel circuits between rat nucleus accumbens and thalamus revealed by retrograde trans-synaptic transport of pseudorabies virus. J Neurosci 17:2143-2167.

Oyster CW (1999) The human eye. Sunderland, MA: Sinauer.

Perez Fontan JJ, Velloff CR (1997) Neuroanatomic organization of the parasympathetic bronchomotor system in developing sheep. Am J Physiol 273:R121-R133.

Petrovskis EA, Timmins J, Gierman T, Post L (1986) Deletions in vaccine strains of pseudorabies virus and their effect on synthesis of glycoprotein gp63. J Virol 60:1166-1169.

Pickard GE (1982) The afferent connections of the suprachiasmatic nucleus of the golden hamster with emphasis on the retinohypothalamic projection. J Comp Neurol 211:65-83.

Pickard GE (1985) Bifurcating axons of retinal ganglion cells terminate in the hypothalamic suprachiasmatic nucleus and the intergeniculate leaflet of the thalamus. Neurosci Lett 55:211-217.

Pickard GE, Card JP, Moore RY, Weeks J (1992) Pseudorabies virus labeling of the suprachiasmatic nucleus and intergeniculate leaflet in the golden hamster. Soc Res Biol Rhythms 3:64.

Pickard GE, Tomlinson CC, Smeraski CA, Wilcox CL, Banfield BW, Enquist LW, Sollars PJ (2001) PRV Bartha infects the suprachiasmatic nucleus in rodents via transneuronal spread through retrograde circuitry. Soc Neurosci Abstr 31:91.

Provencio I, Cooper HM, Foster RG (1998) Retinal projections in mice with inherited retinal degeneration: implications for circadian photoentrainment. J Comp Neurol 395:417-439.

Ranson SW, Magoun HW (1933) The central path of the pupilloconstrictor reflex in response to light. Arch Neurol Psychiatry 30:1193-1204.

Saper CB, Loewy AD, Swanson LW, Cowan WM (1976) Direct hypothalamo-autonomic connections. Brain Res 117:305-312.

Scalia F (1972) The termination of retinal axons in the pretectal region of mammals. J Comp Neurol 145:223-257.

Smith BN, Banfield BW, Smeraski CA, Wilcox CL, Dudek FE, Enquist LW, Pickard GE (2000) Pseudorabies virus expressing enhanced green fluorescent protein: a tool for in vitro electrophysiological analysis of transsynaptically labeled neurons in identified central nervous system circuits. Proc Natl Acad Sci USA 97:9264-9269.

Smith KC (1997) Herpesviral abortion in domestic animals. Vet J 153:253-268.

Strack AM, Loewy AD (1990) Pseudorabies virus: a highly specific transneuronal cell body marker in the sympathetic nervous system. J Neurosci 10:2139-2147.

Swanson LW (1977) Immunohistichemical evidence for a neurophysincontaining autonomic pathway arising in the paraventricular nucleus of the hypothalamus. Brain Res 128:346-353.

Teclemariam-Mesbah R, Kalsbeek A, Pevet P, Buijs RM (1997) Direct vasoactive intestinal polypeptide-containing projection from the suprachiasmatic nucleus to spinal projecting hypothalamic paraventricular neurons. Brain Res 748:71-76.

Tomishima MJ, Enquist LW (2001) A conserved $\alpha$-herpesvirus protein necessary for axonal localization of viral membrane proteins. J Cell Biol 154:741-752.

Ueyama T, Krout KE, Nguyen XV, Karpitskiy V, Kollert A, Mettenleiter TC, Loewy AD (1999) Suprachiasmatic nucleus: a central autonomic clock. Nat Neurosci 2:1051-1053.

Valentino RJ, Kosboth M, Colflesh M, Miselis RR (2000) Transneuronal labeling from the rat distal colon: anatomic evidence for regulation of distal colon function by a pontine corticotropin-releasing factor system. J Comp Neurol 417: 399-414.

Watts AH (1991) The efferent projections of the suprachiasmatic nucleus: anatomical insights into the control of circadian rhythms. In: Suprachiasmatic nucleus: the mind's eye (Klein DC, Moore RY, Reppert SM, eds), pp 77-106. New York: Oxford UP.

Watts AG, Swanson LW, Sanchez-Watts G (1987) Efferent projections of the suprachiasmatic nucleus: I. Studies using anterograde transport of Phaseolus vulgaris leucoagglutinin in the rat. J Comp Neurol 258:204-229.

Whealy ME, Card JP, Robbins AK, Dubin JR, Rziha HJ, Enquist LW (1993) Specific pseudorabies virus infection of the rat visual system requires both gI and gp63 glycoproteins. J Virol 67:3786-3797.

Young MJ, Lund RD (1994) The anatomical substrates subserving the pupillary light reflex in rats: origin of the consensual pupillary response. Neuroscience 62:481-496.

Young MJ, Lund RD (1998). The retinal ganglion cells that drive the pupilloconstrictor response in rats. Brain Res 787:191-202.

Zheng J-Q, Seki M, Hayakawa T, Ito H, Zyo K (1995) Descending projections from the paraventricular hypothalamic nucleus to the spinal cord: anterograde tracing study in the rat. Okajimas Folia Anat Jpn 72:119-136 